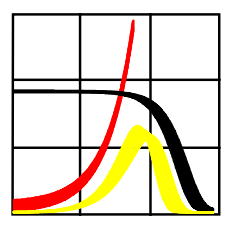

Max-Planck-Institut für demografische Forschung

Max Planck Institute for Demographic Research

Doberaner Strasse 114 - D-18057 Rostock · GERMANY

Tel +49 (0) 3812081 - 0; Fax +49 (0) 3812081 - 202;

http://www.demogr.mpg.de

MPIDR WORKING PAPER WP 1999-004

APRIL 1999

\title{
Reproductive History and Mortality \\ Later in Life: A Comparative Study of \\ England \& Wales and Austria
}

\author{
Gabriele Doblhammer \\ (doblhammer@demogr.mpg.de)
}

This working paper has been approved for release by: James W. Vaupel (jwv@ demogr.mpg.de) Head of the Laboratory of Survival and Longevity.

(C) Copyright is held by the author.

Working papers of the Max Planck Institute for Demographic Research receive only limited review. Views or opinions expressed in working papers are attributable to the author and do not necessarily reflect those of the Institute. 


\author{
Gabriele Doblhammer \\ Max Planck Institute \\ for Demographic Research \\ Doberanerstr. 114 \\ 18057 Rostock
}

Germany

Tel: +493812081 124

Fax: +493812081424

e-mail: doblhammer@demogr.mpg.de

\title{
Reproductive History and Mortality Later in Life
}

A comparative study of England \& Wales and Austria

\author{
Gabriele Doblhammer
}

Max Planck Institute for Demographic Research, Doberanerstr. 114, 18057 Rostock, Germany. E-mail:doblhammer@demogr.mpg.de 


\begin{abstract}
Does a woman's reproductive history influence her life span? This study explores the question on the basis of data from two contemporary female populations: England \& Wales and Austria. It is the first comparative study that investigates the relationship between fertility and mortality late in life. We find similar patterns and age-specific trends of excess mortality in both populations: parity significantly influences longevity, as do both an early and a late birth. These differences in longevity are not explained by differences in educational or family status. The impact of a woman's reproductive history on her life span is minor, however, compared to the influence of her level of education or family status.
\end{abstract}




\section{INTRODUCTION}

Although most deaths in contemporary populations occur at older ages, little isknown about the factors that influence mortality at these ages (Christensen and Vaupel 1996). In a recent paper by Vaupel et al. (1998) the authors write that 'deeper understanding of survival at older ages hinges on intensified research into the interactions between fertility and longevity'. A leading biological theory of the evolution of senescence stresses that resources have to be directed either towards somatic maintenance or towards reproduction (Kirkwood 1977, Kirkwood and Rose 1991). Experiments have indicated that such trade-offs between reproduction and longevity exist in non-human species (e.g. Partridge and Barton 1993). Selection pressures cause species to maximise reproductive success rather than optimising longevity, which, in turn, leads to senescence and ultimately to death.

This study is concerned mainly with the question of whether or not a woman's reproductive history influences her life span. Unlike many non-human species, humans live in complex social and cultural settings that may largely offset any evolutionary trade-off between reproduction and longevity mediated through negative physiological long-term effects. Furthermore, the social and cultural environment may result in a trade-off between fertility and longevity that is unrelated to evolutionary strategy.

Few studies have analysed the influence of fertility on mortality later in life among contemporary human populations (Kitagawa and Hauser 1975, Beral 1985, Kvale et al. 1994, Green et al. 1988, Lund et al. 1990, Friedlander 1996). Most of these studies found a significant negative correlation between parity and longevity. However, results are 
ambiguous concerning nulliparous women. Three of the above studies suggest that childless women experience lower all-cause mortality tharparous women (Beral 1985, Kvale et al., Friedlander et al. 1996). The others conclude that the relationship between parity and mortality is U-shaped: mortality is highest for childless women and women of high parity and lowest for women with two to four children. The only study based on the fertility history of contemporary men (Friedlander 1996) did not find any significant relationship.

The pattern of childbearing has also been investigated Lund et al. 1990, Perls et al. 1997). These studies find positive effects for late first and last births. A few studies have concentrated on historical populations with high fertility regimes. For example, Voland and Engel (1986) analysed the relationship between reproductive history and longevity in historical populations inEast Friesland, Germany, and Le Bourg et al. (1993) studied French immigrants to Quebec. The former find a significant but minor correlation between longevity and both age at last birth and the number of surviving children; the latter authors did not find any relationship between fertility and longevity in their study population. Specifically, they found no connection between early fecundity and longevity. In a recent study of women from aristocratic British families,Westendorp and Kirkwood (1998) find a significant negative correlation between parity and longevity and a positive correlation between age at first childbirth and longevity.

Studies on historical populations are usually based on small data-sets:Voland and Engel use the vital statistics of 811, Le Bourg et al. of 694, and Westendorp and Kirkwood of 1,908 women. Furthermore, these data often lack accuracy concerning dates of birth and death and are subject to underreporting of childbirth. In the case of historical 
populations the analysis of the relationship between fertility and mortality late in life is confined to a highly selected group of women: those who survive the hazards of pregnancies and childbirth to old age. It was often the case that death occurred long before the end of a woman's reproductive phase. In these populations the main cost of reproduction may have lain in the risks of pregnancy and childbirth during the premenopausal phase of life rather than in accumulated negative effects on old age. It has been estimated that from 1780 to 1899 about 152 women per 10,000 live births died in Germany (Imhof 1981). By the mid-twentieth century maternal deaths had been reduced dramatically: in 1946, 32.8 women per 10,000 live births died in Austria and ten years later this figure had dropped to 17.4. Thus, when analysing the impact of fertility on mortality late in life, one mustn't forget that a contemporary female population is far less selected than any historical population.

To avoid the many problems associated with data from historical populations, this article focuses on two contemporary populations for which large data-sets are available: Austria and England \& Wales. The Austrian data-set contains all women aged 50 to 94 who were enumerated in the 1981 census; the English/Welsh data-set is based on a one per cent sample of the 1971 census $^{1}$.

\section{DATA AND METHODS}

Data

This comparative study is based on two different data-sets. The Austrian cross-sectional data-set links death and census records. The census records provide information not only about socio-economic and socio-demographic characteristics but also about the complete 
reproductive history of women, including the exact age of the mother up to the fourth birth. The 1981 census of the Austrian population enumerated 1,254,153 women between the ages of 50 and 94 who had ever been married; in a further step, the death records of 35,234 women who died within one year after the census were then linked to the census records (Doblhammer 1996).

The Longitudinal Study of the Office for National Statistics (ONS), which is based on a one per cent sample of the population of England and Wales, consists of cohort data (Hattersley and Creeser 1995). It includes linked census and vital events data on 56,164 ever-married women counted in the 1971 census who were followed until the end of 1996. At baseline, the age range runs from 40 to 59 years. By the end of 1995 these women had reached a maximum age of 85 years. By the end of the observation period, 16,941 women had died.

Both populations exhibit low fertility. A relatively large proportion of the evermarried women remained childless (18.5\% in Austria, $13.2 \%$ in England \& Wales), partly because of the two world wars in the first half of the century; the majority had one or two children (49.3\% in Austria, 55.1\% in England \& Wales). The birth of the first child usually occurred after age 20: only 12\% of Austrian mothers and 7\% of women in England and Wales had their first child before age 20. Only a small minority oparous women in England and Wales gave birth after age forty (8\%). The Austrian data-set contains a mother's age at birth only up to the fourth child: among parous women $7 \%$ had at least one child after age forty; $12 \%$ had five and more children, but their fourth child was born before age forty. 
Methods

The mortality of the female Austrian population was analysed by applying multivariate logistic regression models in the form of:

$$
\pi(y)=\frac{\exp \left(\beta_{0}+\sum_{i} \beta_{i} y_{i}\right)}{1+\exp \left(\beta_{0}+\sum_{i} \beta_{i} y_{i}\right)}=E(X \mid y)
$$

The dependent variable takes the value 'one' when death occurred within one year after the census and 'zero' otherwise. The covariates $\mathrm{y}_{\mathrm{i}}$ are the characteristics 'education', 'family status at the time of the census', 'parity', 'first birth before age 20', and 'at least one birth at ages 40+'. The results are controlled for age, which is defined in five-year age groups. All covariates are defined as indicator variables which take the value 'one' if a characteristic applies to an individual and 'zero' otherwise. Age-specific odds ratios were estimated by introducing an interaction term into the model between the age and the respective fertility pattern. This article refers to the exponents of the parameters of the logistic regression models as relative mortality risks.

In the English and Welsh data-set the force of mortality at age $\mathrm{x}$ was estimated by fitting the Gompertz function 


$$
\begin{aligned}
& \mu(x)=a \exp (\beta x), \\
& \text { with } a=\exp \left(\alpha_{0}+\sum_{i} \alpha_{i} y_{i}\right)
\end{aligned}
$$

'Parity', 'first birth before age 20', 'at least one birth after age forty', and 'educational status' are treated as indicator variables $y_{i}$ which take the value 'one' if a characteristic applies to a women and 'zero' otherwise. The parameters $\alpha$ and $\beta$ were estimated by maximising the likelihood function (see, for exampleKalbfleisch andPrentice 1980). The relative differences in the age-specific hazard functions of women of different fertility patterns were estimated by applying the Kaplan-Meier method (1958); the hazard functions were smoothed by calculating weighted averages of adjacent years. Because the age at baseline ranges from 40 to 59 years, all calculations were corrected for left truncation by specifying the likelihood function conditional on the age at baseline.

\section{RESUlTS}

Table 4 here

This study shows that there exists a significant relationship between parity and mortality late in life in both populations (Table 4): childless women and women of higher parities experience higher mortality risks later in life than women with one or two children. The extent of excess mortality is also comparable. In the data-set for England and Wales, the excess mortality of childless women compared to women with one or two children is $15 \%$; in the Austrian data-set the difference between childless women and mothers with exactly two children is also $15 \%$. The excess mortality of women with at least three children is 
seven per cent in England and Wales. The Austrian data reveal that women experience significant excess mortality from the fourth child on. The excess mortality of women with four children is six per cent and of women with five or more children, ten per cent. This relationship is independent of educational and family status. In the Austrian data-set the relationship persists even after correction for other social characteristics Doblhammer 1996).

Table 5 here

The relationship between parity and mortality late in life is not the result of the timing of births (Table 5). Among parous women it still persists even after correction for age at first birth and for giving birth after age forty. A birth before age 20 significantly increases the mortality risk late in life in both populations but in the English/Welsh data-set excess mortality is nearly three times as high (26\%) as in the Austrian data-set (9\%). A birth after age forty reduces the risk in both populations by five per cent. However, for English and Welsh women this reduction is only significant at the 90 per cent level.

Figures 1 - 4 here

Age-specific hazard rates reveal similar trends in both populations. For English and Welsh women Figures 1 to 4 contain the ratios of the age-specific hazard rates; for Austrian women, they contain relative mortality risks by age. The excess mortality of childless 
women compared to women with one or two children peaks before age sixty and then decreases with age. In the Austrian data-set the excess mortality of women with three or more children increases with age. At age fifty their mortality is lower than that of all other women; at older ages, however, it is even higher than the mortality of childless women. In the English/Welsh data-set excess mortality peaks at age 75 and decreases for older ages.

The less favourable mortality situation of women who gave birth before their $2 \sigma^{\circ}$ birthday (early mothers) persists in all age groups. Both data-sets show a declining trend with age but in the English/Welsh data-set the decline does not start until age 66. Thus, the difference between Austria and England \& Wales in the excess mortality of early mothers results from the difference between the ages 60 and 82 .

The age-specific mortality risks of late mothers (at least one birth at age 40+) compared to young mothers (last birth before age 40) differ substantially from the previously described risk patterns. In general, the mortality of late mothers is higher than that of young mothers, with the exception of the age range from 60 to 70 .

\section{DISCUSSION}

The present study evaluates women's mortality risks late in life in relation to their reproductive history. The results are based on two data-sets: first, a linked data-set that combines the information from the 1981 census of the Austrian population with the information from the death certificates of those women who died during the year immediately following the census; second, the England and Wales 1\% Longitudinal Study, which includes the fertility history of one per cent of the ever-married women enumerated in the 1971 census. These women were followed until the end of 1996. Unlike previous 
studies, which were usually based on rather small samples from one population only, this study analyses the costs and benefits of reproduction on the basis of 1,254,153 Austrian and 56,164 English and Welsh women, with 35,234 and 16,941 deaths, respectively.

This analysis is based on contemporary populations for three reasons. First, contemporary post-menopausal women are a far less selected group than was previously the case. For historical populations the costs of reproduction lay mainly not in old age, but in the hazards of pregnancies and childbirth during the reproductive period. Thus, those women who survived beyond menopause may have differed only marginally in the impact their reproductive history had on their mortality at old ages. One disadvantage of studying a totally contemporary population is that modern hormonal replacement therapies and hormonal contraceptives may well influence the relationship between reproduction and longevity. In this regard our Austrian study-population is ideal: it consists of women between the ages of 50 and 94 at the time of the census in 1981. It is unlikely that these women had ever taken hormones endogenously (Kopera 1991). The data-set for England and Wales used in this study differs in this respect since it includes younger cohorts (Moorhead et al. 1997, Townsend 1998). The second reason for choosing contemporary populations is that the reporting of birth and death dates and of the number of children is more accurate than for historical populations. The third reason is the availability of large data-sets that contain both the fertility history and the survival of each individual.

Some authors of studies on historical populations (see, for example, Westendorp and Kirkwood 1998) have speculated that the relationship between fertility and mortality late in life be seen - if at all - only in high fertility populations. This study reveals, however, that a significant relationship exists in contemporary populations as well. For 
both populations in our study similar patterns of excess mortality are found.Nulliparous and high-parity women experience significant excess mortality compared to women with one or two children; the mortality of women who gave birth before their $20^{\text {th }}$ birthday exceeds that of women whose first birth was after age 20; late mothers experience lower mortality than young mothers.

The excess mortality of high-parity women appears to support the hypothesis of a physiological trade-off between reproduction and longevity. Further evidence, concerning males, is however required: if the relationship is the result of the physiological long-term effects of childbearing per se, then the pattern should be found in females only.

Unfortunately, neither of the two data-sets provides the fertility history of men. The only study to date that analyses the impact of parity on longevity for a contemporary male population did not find any significant relationshipF(riedlander 1996). A study by Christensen et al. (1998) revealed that the number of remaining teeth in old age is negatively correlated with parity among women but not among men. These two studies suggest the existence of physiological factors. In contrast, Westendorp and Kirkwood (1998) found a significant pattern for both females and males in a historical population of aristocratic British families.

The Austrian data-set suggests that the mortality disadvantage of women of higher parities increases with age. This is most interesting in light of research on carearrangements for frail elderly people. It was found that the likelihood of receiving any help from children increases with the number of children \$pitze and Logan 1990), and it is not just the number of caregivers that increases with the number of living children but also the total hours of care (Wolf et al. 1997). Thus, in terms of receiving care at older ages a large 
number of children is favourable. But this advantage is not reflected in the age-specific mortality risks of Austrian women.

In the Austrian data-set age and cohort effects are confounded. And in the English and Welsh data-set the excess mortality of high parity women decreases from age 75 onwards; at ages $80+$ their mortality is even lower than that of mothers with one or two children.

One could argue that the mortality disadvantage late in life is the result of social hardships that are correlated with a large number of children. Education among the elderly is a very crude measure to adjust for social differences. However, we know from studies on socio-economic differentials in mortality Martelin 1994, Doblhammer 1996) that the differences decrease with advancing age, which is contrary to the increase in excess mortality of high-parity women found in this study. Furthermore, in the Austrian data-set the relationship persists even after correction for factors like occupational status, quality of housing, and whether a woman was predominantly economically active or a housewife.

This study finds significant excess mortality for childless women compared to women with one or two children. The excess mortality of childless women has been repeatedly attributed to health selection. The argument is that women remain childless because they are less healthy and their excess mortality is independent of their parity status. The comparative analysis of the two study populations reveals that, despite the fact that the proportion of childless women in England and Wales is substantially lower than in Austria, excess mortality is similar. This appears to contradict the assumption that health selection plays a dominant role: the larger the proportion of childless women, the more 
likely it is that these women remained childless not only because of their health but also due to spinsterhood or widowhood.

The proportion of English and Welsh women who gave birth before ge 20 is only half that of Austrian women, and yet their excess mortality is nearly three times that of comparable Austrian women. This difference is due to the fact that in Austria excess mortality decreases with age, whereas in England and Wales it remains at a high level. One possible explanation may be that the negative social circumstances that may have been related to giving birth comparatively early in life were more severe in England and Wales than in Austria, where the phenomenon was more widespread.

This study finds a positive relationship between giving birth after age forty and longevity, which is consistent with the findings of two previous studies Perl et al. 1997, Voland and Engel 1986). In addition, the Austrian data suggest that the longevity advantage of late mothers results from a reduction in the mortality risk from circulatory diseases, although at the same time their risk of breast cancer is significantly increased (Doblhammer 1998). One possible explanation may be that giving birth after age forty is a biological marker that these women have always aged at a slower pace and that their menopause occurred comparatively late (Perls et al. 1997). A variety of studies (Bromberg et al. 1997, Stanford et al. 1987, Whelan et al. 1990) demonstrate that, along with other factors, the age at menopause correlates positively with parity in general and with whether a woman ever had a live birth inparticluar. Bromberg et al. found that the median age at menopause is 1.3 years lower for women who never gave birth. Snowdon et al. (1989, 1990) show that the mortality risk for women who had their natural menopause before the age of forty is nearly twice as high as for those who experienced menopause at age 50-54. 
It could be that the positive impact of late menopause results from the extended period of endogenous oestrogen production. From the endocrine point of view, menopause is considered a permanent state of oestrogen-deficiency. A variety of studies have shown that post-menopausal oestrogen therapies, which restore the pre-menopausal endocrine milieu, are associated with a reduced risk of heart disease Paganini-Hill 1997, Prelevic 1997), osteoporosis (Seeman 1997), and Alzheimer's disease (Kawas et al. 1997).

On the negative side, however, a number of studies also suggest the possibility of an increase in the risk of breast cancer due to oestrogen therapies (Paganini-Hill 1997, Law et al. 1996). A positive correlation between age at natural menopause and breast cancer was found by Heck and Pamuk (1997).

Is giving birth after age forty an indicator for late menopause? Probably not, because it is only in recent cohorts that we see a continuous decline in the upper age of childbearing (Campbell and Wood 1994) while the average age at menopause seems to remain more or less constant over time. Thus, it is plausible to assume that a large proportion of women whose last birth was before age forty did not give birth later for social rather than for physiological reasons. It may therefore well be that the social factors that encourage a late birth are the driving forces behind the increase in the longevity of late mothers.

Even if it is the case that a late birth indicates late menopause, this does not necessarily imply that late mothers have always aged at a slower pace. An extended period of childbearing and child-raising could be associated with healthy behaviour. For example, smoking is one of the well-established determinants of age at menopause: research shows 
that cigarette smoking decreases the age at menopause by one to two years (e.g. McKinlay et al. 1985). Thus, late mothers may indeed experience menopause at an age higher than that of young mothers but this could be due to healthy behaviour rather than to a lower rate of ageing.

\section{CONCLUSION}

The results of this study are consistent with the hypothesis that reproduction and longevity are closely related. However, differences in reproductive history do not explain much of the large variability in longevity. The influence of reproductive history on longevity, although statistically significant, is minor compared to differences in longevity stemming from environmental factors such as the level of education or family status. Among the different patterns of childbearing, giving birth early in life has the largest impact on longevity. It is also the only fertility characteristic that differs between England \& Wales and Austria in terms of the extent of excess mortality. The question of whether the observed relationship between reproduction and longevity among women indicates that there is a physiological trade-off between reproduction and longevity or whether it is the result of social and cultural factors cannot be answered decisively. For humans, insights will be gained by conducting research on the basis of individual data that contain full life histories rather than just fertility histories. The analysis of the relationship between fertility and longevity among men will also shed light on this question. 


\section{REFERENCES}

Beral, V. 1985. "Long term effects of childbearing on health", Journal of Epidemiology and Community Health 39: 343-346.

Bromberg, J. T. et al. 1997. "Prospective Study of the Determinants of Age at Menopause", American Journal of Epidemiology 145(2): 124-133.

Campbell, K.L. and J.W. Wood. 1994. "Introduction: What is Human Reproductive Ecology, and Why should we care about studying it?", in Campbell K.L and Wood J.W. (eds.) "Human Reproductive Ecology. Interactions of Environment, Fertility, and Behavior. "Annals of the New York Academy of Sciences709: New York.

Christensen, K. et al. 1998. “A tooth per child?”, The Lancet 352:204.

Christensen, K. and J. W. Vaupel. 1996. "Determinants of Longevity: genetic, environmental and medical factors",Journal of Internal Medicine 240: 333-341.

Doblhammer, G. 1996. Socioeconomic Differentials in Austrian Adult Mortality. University of Vienna: unpublished dissertation.

Doblhammer, G. 1998. Reproductive History and Mortality Later in Life: unpublished working paper.

Partridge, L. and Barton N.H.. 1993. "Optimality, mutation and the evolution of ageing", Nature 338: 305-311.

Friedlander, N.J. 1996. "The Relation of Lifetime Reproduction to Survivorship in Women and Men: A Prospective Study”, American Journal of Human Biology 8: 771783.

Green, A., et al. 1988. "Mortality in women in relation to their childbearing history", British Medical Journal 297: 391-395. 
Hattersley, L. and R. Creeser. 1995. Longitudinal Study 1971-1991: History, organisation and quality of data. Series LS No.7. HMSO, London.

Heck, K. E. and E. R. Pamuck. 1997. "Explaining the Relation between Education and Postmenopausal Breast Cancer", American Journal of Epidemiology 145(4): 366-372.

Imhof, A. E. 1981. "Unterschiedliche Säuglingssterblichkeit in Deutschland, 18.-20. Jahrhundert - Warum?", Zeitschrift für Bevölkerungswissenschaft 7: 343-382.

Kalbfleisch, J.D. and R.L. Prentice. 1980. The Statistical Analysis of Failure Time Data. New York:Wiley.

Kaplan E.L. and P. Meier. 1958. "Nonparametric Estimation from Incomplete Observations", Journal of the American Statistical Association 53: 457-481.

Kawas, C. et al. 1997. "A prospective study of estrogen replacement therapy and the risk of developing Alzheimer's disease: The Baltimore Longitudinal Study of Aging", Neurology 48/6: 1517-1521.

Kirchengast, S. 1994. “Anthropological Aspects of the Age at Menopause”, Homo 44(3): 263-277.

Kirkwood, T.B.L. 1977. "Evolution of ageing”, Nature 270:301-302.

Kirkwood T.B.L. and M.R. Rose. 1991. "Evolution of senescence: late survival sacrificed for reproduction", Phil. Trans. R. Soc. Lond. B 332: 15-24.

Kitagawa, E. and P. Hauser. 1973. Differential Mortality in the United States: A Study in Socioeconomic Epidemiology. Cambridge, Mass.: Harvard University Press.

Kopera, H. 1991. "History of the hormonal treatment of climacteric disease symptoms", Wiener Medizinische Wochenschrift 141(15):346-348. 
Kvale, G. et al. 1994. "Parity in relation to mortality and cancer incidence: A prospective study of Norwegian women", International Journal of Epidemiology 23: 691-699.

Law, T. M. et al. 1996. "Breast cancer in elderly women: Presentation, survival, and treatment options", Surgical Clinics of North America 76/2: 289-308.

Le Bourg, E. et al. 1993. "Reproductive Life of French-Canadians in the $17-18^{\text {th }}$ Centuries: A Search for a Trade-off between early Fecundity and Longevity", Experimental Gerontology 28: 217-232.

Lund, E. et al. 1990. "Pattern of childbearing and mortality in married women - A national prospective study from Norway", Journal of Epidemiology and Community Health 44: 237-240.

Martelin, T. 1994. "Differential mortality at older ages.Sociodemographic mortality differences among the Finnish elderly",Publications of the Finnish Demographic Society

Vol. 16.

McKinley, S.M., N.L. Bifano and J.B. McKinley. 1985. "Smoking at age at menopause in women”, Ann Intern Med 103: 350-356.

Moorhead, T., P. Hannaford and M. Warskyj. 1997. "Prevalence and characteristics associated with use of hormone replacement therapy in Britain”,British Journal of Obstetrics and Gynaecology 104:290-297.

Paganini-Hill, A. 1996. "Estrogen replacement therapy in the elderly", Zentralblatt für Gynäkologie 118/5: 255-261.

Perls, T. T., L. Alpert, and R. C. Fretts. 1997. "Middle aged mothers live longer", Nature 389: 133. 
Prelevic, G. M. 1997. "New developments in postmenopausal hormone replacement therapy",Curr Opin Obstet Gynecol 9(3): 207-212.

Seeman, E. 1997. "Osteoporosis: trials and tribulations”, American Journal of Medicine 103(2A): 74S-87S.

Snowdon, D. A. et al. 1989. "Is early natural menopause a biological marker of health and aging?", American Journal of Public Health 79: 709-14.

Snowdon, D. A. 1990. "Early natural menopause and the duration of postmenopausal life. Findings from a mathematical model of life expectancy", Journal of the American Geriatric Society 38(4): 402-408.

Spitze, G. and J. Logan. 1990. "Sons, daughters, and intergenerational support", Journal of Marriage and the Family 52: 420-430.

Stanford, J. L. et al. 1987. "Factors influencing the age at menopause", Journal of Chronical Diseases 40: 995-1002.

Townsend, J. 1998. "Hormone replacement therapy: assessment of present use, costs, and trends", British Journal of General Practice 48: 955-8.

Vaupel, J. W. et al. 1998. "Biodemographic Trajectories of Longevity", Science 280:855860.

Voland, E. and C. Engel. 1986. "Ist das postmenopausale Sterbealter Variable einer fitness-maximierenden Reproduktionsstrategie?", Anthropologischer Anzeiger 44(1):1934.

Westendorp, R.G. and T.B.L Kirkwood. 1998. "Human longevity at the cost of reproductive success", Nature 396:743-746. 
Whelan, E. A. et al. 1990. "Menstrual and reproductive characteristics and age at natural menopause", American Journal of Epidemiology 131: 625-32.

Wolf, D. A, V. Freedman, and B. J. Soldo. 1997. "The division of familylabor: care for elderly parents", The Journals of Gerontology Series B, Psychological Science and Social Science 52: 102-109. 
Table 1: Number of deaths and censored by parity for England \& Wales and Austria

\begin{tabular}{|c|c|c|c|c|c|c|}
\hline Parity & Cens & red & & & Total at & aseline \\
\hline England and Wales $^{1}$ & Count & $\%$ & Count & $\%$ & Count & $\%$ \\
\hline 0 & 4,810 & 12.4 & 2,519 & 14.9 & 7,329 & 13.2 \\
\hline $1+2$ & 21,512 & 55.5 & 9,171 & 54.1 & 30,683 & 55.1 \\
\hline $3+$ & 12,437 & 32.1 & 5,251 & 31.0 & 17,688 & 31.8 \\
\hline all & 38,759 & 100.0 & 16,941 & 100.0 & 55,700 & 100.0 \\
\hline Austria $^{2}$ & & & & & & \\
\hline 0 & 223,496 & 18.3 & 8,796 & 25.0 & 232,292 & 18.5 \\
\hline 1 & 304,157 & 25.0 & 8,410 & 23.9 & 312,567 & 24.9 \\
\hline 2 & 298,354 & 24.5 & 7,190 & 20.4 & 305,544 & 24.4 \\
\hline 3 & 173,043 & 14.2 & 4,308 & 12.2 & 177,351 & 14.1 \\
\hline 4 & 96,512 & 7.9 & 2,586 & 7.3 & 99,098 & 7.9 \\
\hline $5+$ & 123,357 & 10.1 & 3,944 & 11.2 & 127,301 & 10.2 \\
\hline all & $1.218,919$ & 100.0 & 35,234 & 100.0 & $1.254,153$ & 100.0 \\
\hline
\end{tabular}


Table 2: Number of deaths and censored by age at first birth for England \& Wales and Austria

\begin{tabular}{lcccccc}
\hline Age at first birth & \multicolumn{2}{c}{ Censored } & \multicolumn{2}{c}{ Deaths } & \multicolumn{2}{c}{ Total at baseline } \\
\hline England and Wales ${ }^{1}$ & Count & $\%$ & Count & $\%$ & Count & $\%$ \\
\cline { 2 - 7 }$<20$ & 2,052 & 5.3 & 1,065 & 6.3 & 3,117 & 5.6 \\
$20+$ & 31,897 & 82.3 & 13,357 & 78.8 & 45,254 & 81.2 \\
parity 0 & 4,810 & 12.4 & 2,519 & 14.9 & 7,329 & 13.2 \\
all & 38,759 & 100.0 & 16,941 & 100.0 & 55,700 & 100.0 \\
Austria & & & & & & \\
$<20$ & 122,508 & 10.1 & 3,147 & 8.9 & 125,655 & 10.0 \\
$20+$ & 872,915 & 71.6 & 23,291 & 66.1 & 896,206 & 71.5 \\
parity 0 & 223,496 & 18.3 & 8,796 & 25.0 & 232,292 & 18.5 \\
all & $1.218,919$ & 100.0 & 35,234 & 100.0 & $1.254,153$ & 100.0 \\
\hline
\end{tabular}

${ }^{1}$ Source: ONS Longitudinal Study - Crown Copyright

${ }^{2}$ Source: Österreichisches Statistisches Zentralamt 
Table 3: Number of deaths and censored by birth after age 40 for England \& Wales and Austria

\begin{tabular}{lcccccc}
\hline Birth at ages 40+ & \multicolumn{2}{c}{ Censored } & \multicolumn{2}{c}{ Deaths } & \multicolumn{2}{c}{ Total at baseline } \\
\hline England and Wales & Count & $\%$ & Count & $\%$ & Count & $\%$ \\
\cline { 2 - 7 } yes & 2,538 & 6.5 & 1,151 & 6.8 & 3,689 & 6.6 \\
no & 31,411 & 81.0 & 13,271 & 78.3 & 44,682 & 80.2 \\
parity 0 & 4,810 & 12.4 & 2,519 & 14.9 & 7,329 & 13.2 \\
all & 38,759 & 100.0 & 16,941 & 100.0 & 55,700 & 100.0 \\
Austria & & & & & & \\
yes & & & & & & \\
no & 65,676 & 5.4 & 1,980 & 5.6 & 67,656 & 5.4 \\
$4^{\text {th }}$ birth under age 40, & 117,998 & 9.7 & 3,733 & 10.6 & 121,731 & 9.7 \\
age at birth 5+ & & & & & & \\
unknown & & & & & & \\
parity 0 & & & & & & \\
all & 223,496 & 18.3 & 8,796 & 25.0 & 232,292 & 18.5 \\
\hline
\end{tabular}

${ }^{1}$ Source: ONS Longitudinal Study - Crown Copyright

${ }^{2}$ Source: Österreichisches Statistisches Zentralamt 
Table 4: Relative mortality risks (RMR) by parity: Austria ages 50-94, England \& Wales ages 50-85.

\begin{tabular}{|c|c|c|c|c|c|}
\hline \multicolumn{3}{|c|}{ England and Wales } & \multicolumn{3}{|c|}{ Austria } \\
\hline \multicolumn{3}{|c|}{ RMR } & \multicolumn{3}{|c|}{ RMR } \\
\hline Parity & & & Parity & & \\
\hline 0 & 1.15 & $* * *$ & 0 & 1.15 & $* * *$ \\
\hline $1+2(\mathrm{RG})$ & 1.00 & & 1 & 1.01 & \\
\hline \multirow[t]{4}{*}{$3+$} & 1.07 & $* * *$ & $2(\mathrm{RG})$ & 1.00 & \\
\hline & & & 3 & 1.02 & \\
\hline & & & 4 & 1.06 & $* *$ \\
\hline & & & $5+$ & 1.10 & $* * *$ \\
\hline Education & & & Education & & \\
\hline high (RG) & 1.00 & & high (RG) & 1.00 & \\
\hline medium & 1.36 & $* * *$ & medium & 1.16 & $* * *$ \\
\hline \multirow[t]{5}{*}{ basic } & 1.71 & $* * *$ & basic & 1.38 & $* * *$ \\
\hline & & & Family status & & \\
\hline & & & married $(\mathrm{RG})$ & 1.00 & \\
\hline & & & widowed & 1.27 & $* * *$ \\
\hline & & & divorced & 1.39 & $* * *$ \\
\hline \multicolumn{6}{|c|}{$* * *$ significant at the 99 per cent level } \\
\hline \multicolumn{6}{|c|}{$* *$ significant at the 95 per cent level } \\
\hline
\end{tabular}


Table 5: Relative mortality risk (RMR) by parity, age at first birth and birth after age 40:

Austria ages 50-94, England \& Wales ages 50-85.

\begin{tabular}{|c|c|c|c|c|c|}
\hline \multicolumn{3}{|c|}{ England and Wales } & \multicolumn{3}{|l|}{ Austria } \\
\hline & RMR & & & RMR & \\
\hline Parity & & & Parity & & \\
\hline $1+2(\mathrm{RG})$ & 1.00 & & 1 & 1.01 & \\
\hline \multirow[t]{3}{*}{$3+$} & 1.06 & $* * *$ & $2(\mathrm{RG})$ & 1.00 & \\
\hline & & & 3 & 1.02 & \\
\hline & & & $4+$ & 1.06 & $* * *$ \\
\hline Age at first birth & & & Age at first birth & & \\
\hline$<20$ & 1.26 & $* * *$ & $<20$ & 1.09 & $* * *$ \\
\hline $20+(\mathrm{RG})$ & 1.00 & & $20+(\mathrm{RG})$ & 1.00 & \\
\hline Birth above age 40 & & & Birth above age 40 & & \\
\hline no (RG) & 1.00 & & no $(\mathrm{RG})$ & 1.00 & \\
\hline \multirow[t]{2}{*}{ yes } & 0.95 & $*$ & yes & 0.95 & $* *$ \\
\hline & & & $\begin{array}{l}4^{\text {th }} \text { birth under } 40 \text {, age at } \\
\text { birth } 5+\text { unknown }\end{array}$ & 1.09 & \\
\hline Education & & & Education & & \\
\hline high (RG) & 1.00 & & high (RG) & 1.00 & \\
\hline medium & 1.39 & $* * *$ & medium & 1.22 & $* * *$ \\
\hline \multirow[t]{5}{*}{ low } & 1.74 & $* * *$ & low & 1.43 & $* * *$ \\
\hline & & & Family status & & \\
\hline & & & married (RG) & 1.00 & \\
\hline & & & widowed & 1.24 & $* * *$ \\
\hline & & & divorced & 1.36 & $* * *$ \\
\hline
\end{tabular}

*** significant at the 99 per cent level

** significant at the 95 per cent level

* significant at the 90 per cent level 
RG..reference group 
Figure 1: Age-specific mortality ratios of childless women to women with one or two children: Austria ages 50-94, England \& Wales ages 50-85.

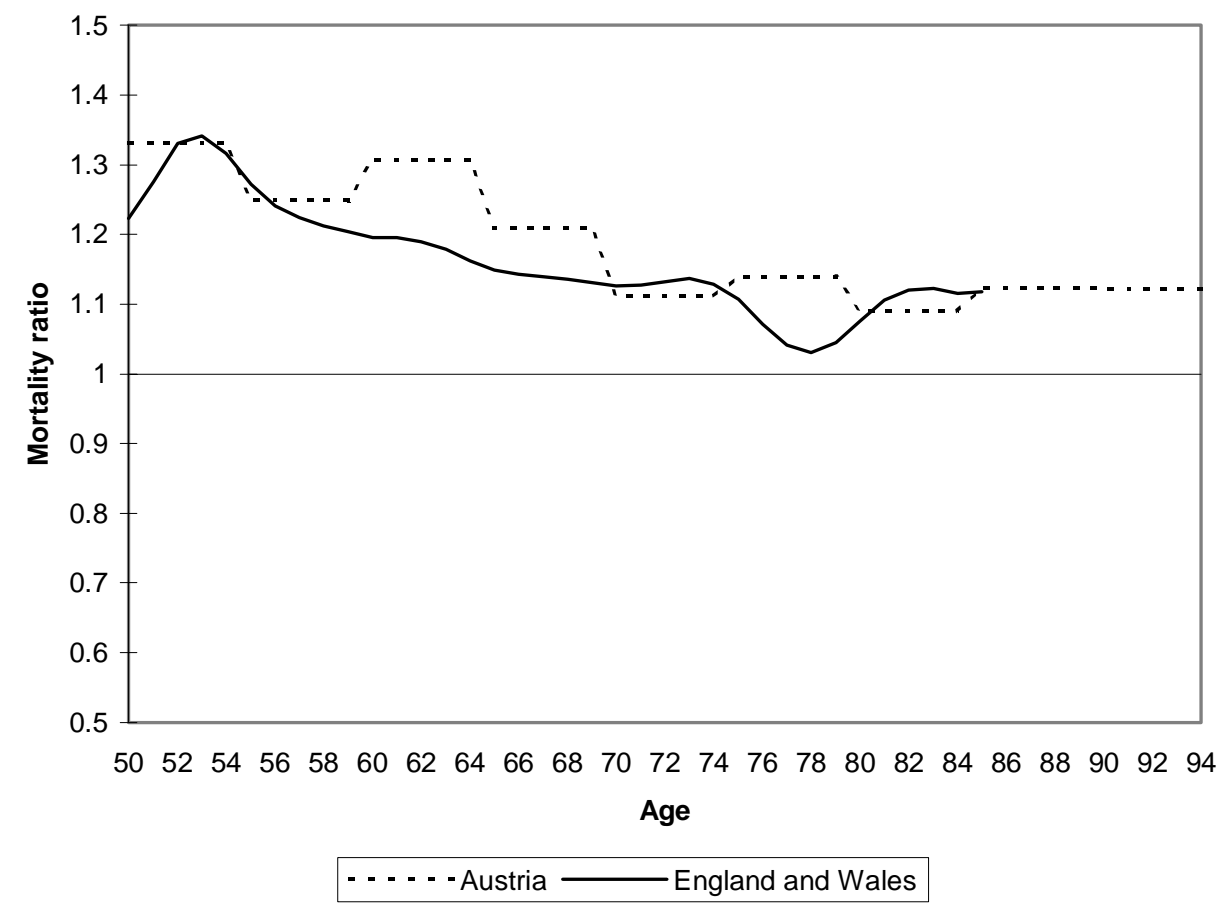


Figure 2: Age-specific mortality ratios of women with three and more children to women with one or two children: Austria ages 50-94, England \& Wales ages 50-85.

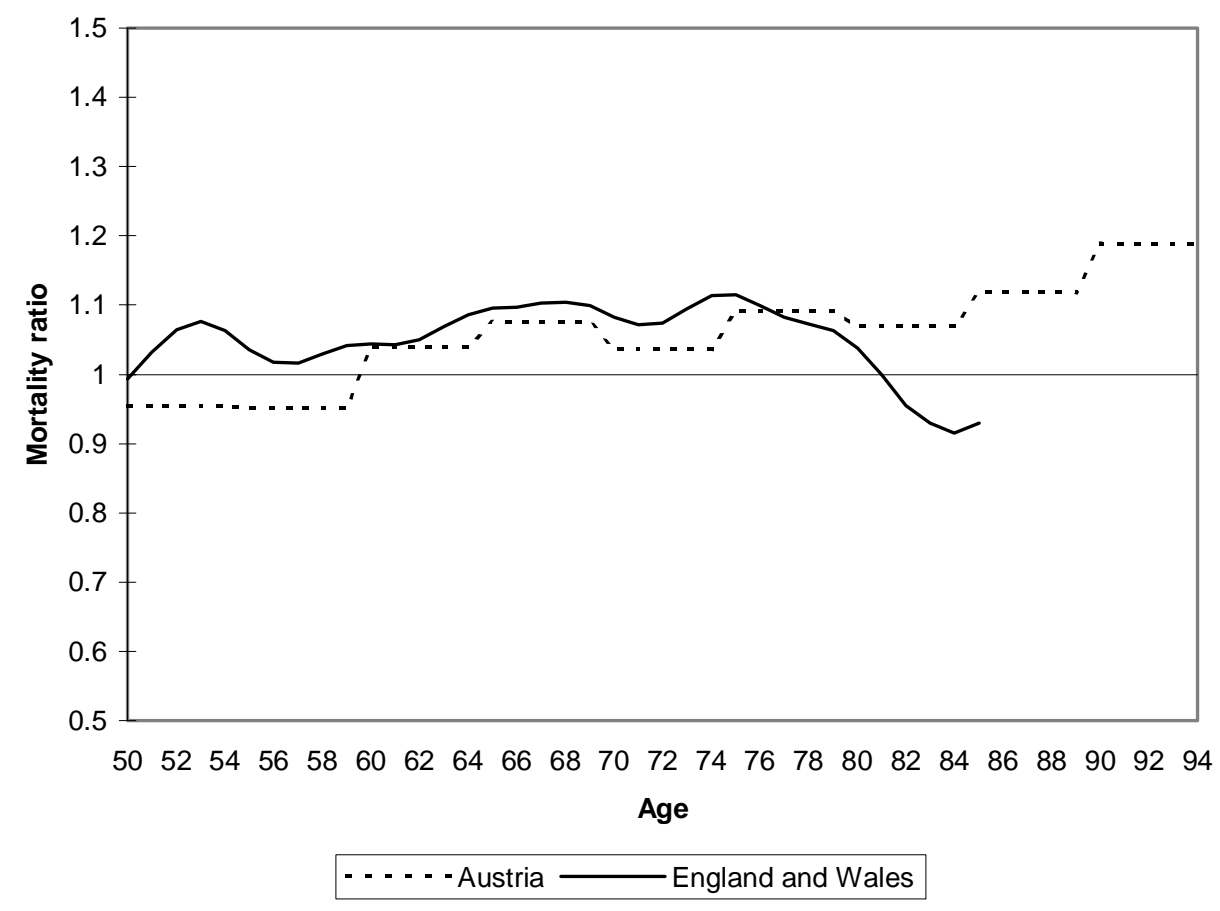


Figure 3: Age-specific mortality ratios of women who gave birth before age 20 to women whose first birth was after age 20: Austria ages 50-94, England \& Wales ages 50-85.

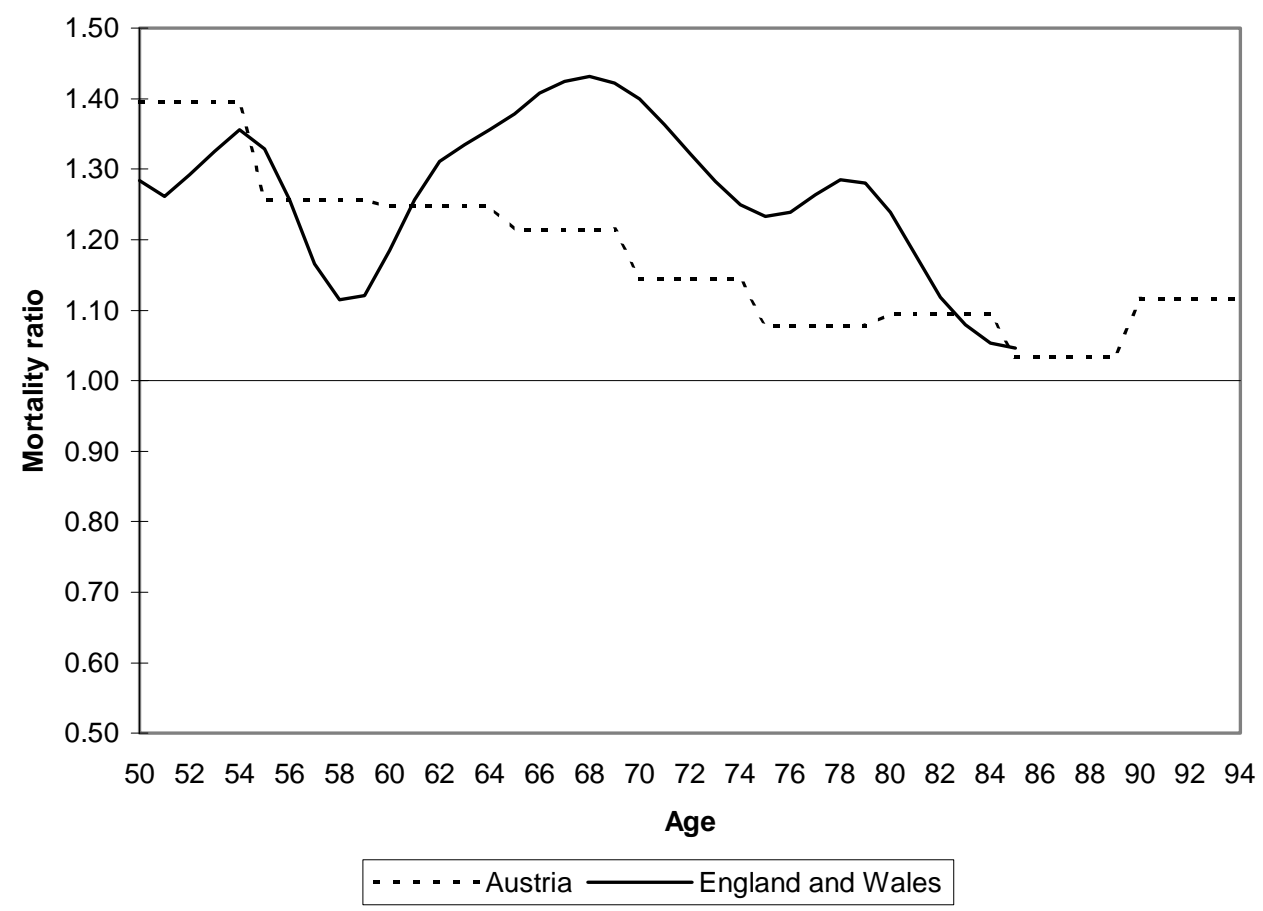


Figure 4: Age specific mortality ratios of women who gave birth after age 40 to women whose last birth was before age 40: Austria ages 50-94, England \& Wales ages 50-85.

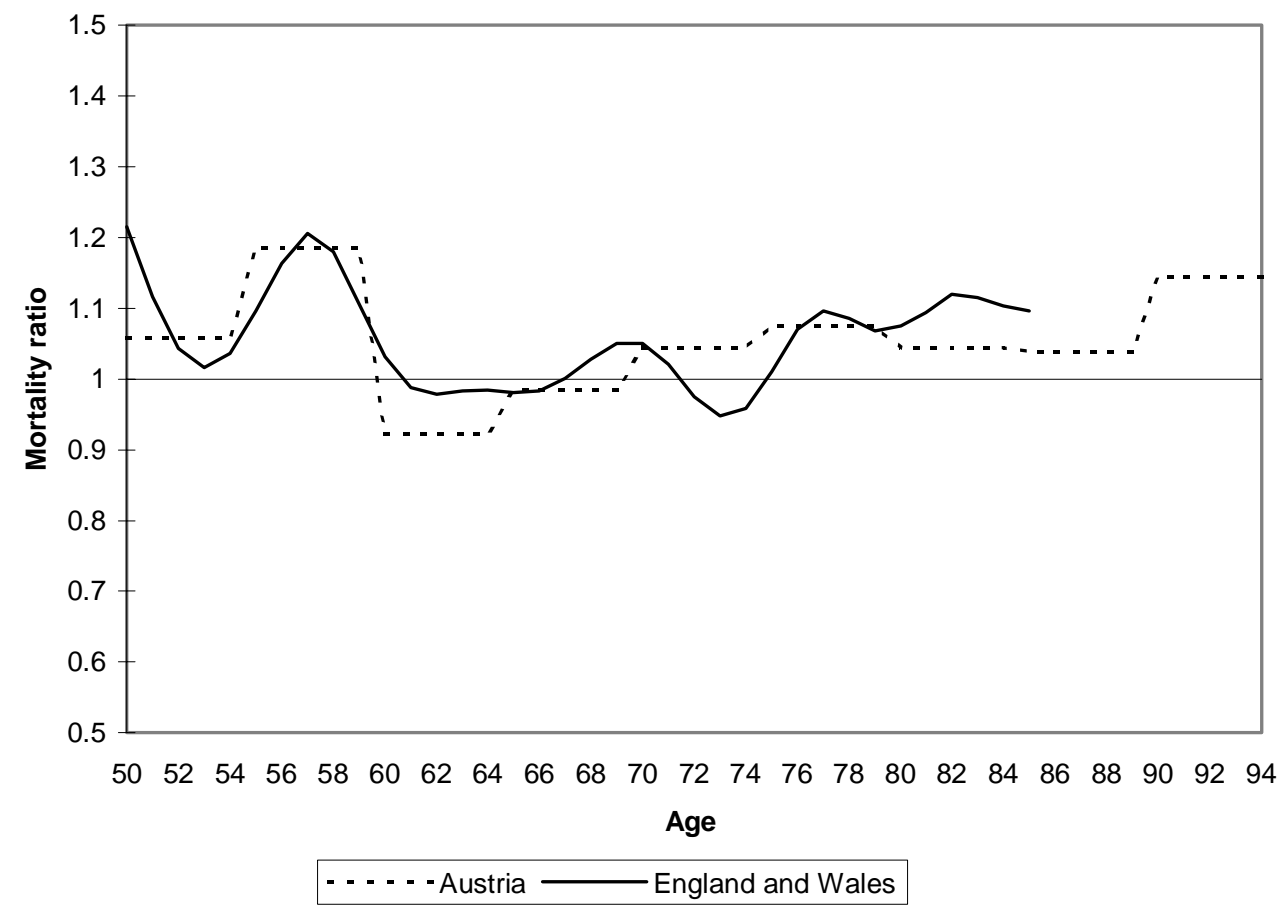




\section{NOTES}

${ }^{1}$ I would like to thank the Office for National Statistics (ONS) for allowing the use of the ONS Longitudinal Study. The views expressed are not necessarily those of ONS.

${ }^{2}$ Education is divided into three groups: basic, medium and high. In the English and Welsh data-set medium education refers to women with diplomas or A level (3,757 women); high education to women who hold a degree (513 women). In the Austrian data-set medium education refers to women with lower secondary education and apprenticeship $(262,123)$; high education to women with upper secondary or tertiary education $(58,270)$.

${ }^{3} \mu(x)$ is approximated by the difference in the cumulated hazard function $\mathrm{H}(\mathrm{x})-\mathrm{H}(\mathrm{x}-1)$. The smoothed value $\hat{\mu}(x)$ is calculated by applying the formula $\widehat{\mu}(x)=\frac{(3 * \mu(x)+2 * \mu(x+1)+2 * \mu(x-1)+\mu(x-2)+\mu(x+2))}{9}$

${ }^{4}$ This is contrary to the hypothesis formulated by Westendorp and Kirkwood (1998); they assume that a similar relationship in males and females supports a biological explanation. This might be correct for some non-human species. However, in the context of research on humans a similar pattern for males and females is a strong indication for the existence of social factors. 